\title{
AVALIAÇÃO DOS DANOS RELACIONADOS AO TRABALHO ENTRE TÉCNICOS-ADMINISTRATIVOS DE UMA INSTITUIÇÃO PÚBLICA DE ENSINO SUPERIOR NA PARAÍBA
}

\author{
EVALUATION OF TECHNICAL ADMINISTRATIVE WORK DAMAGES \\ OF A PUBLICHIGHER EDUCATION INSTITUTION IN PARAÍBA
}

\author{
Letícia de Macêdo Nóbrega Aires ${ }^{1}$ \\ Clésia Oliveira Pachú ${ }^{2}$ \\ Maria do Socorro Rocha Melo Peixoto ${ }^{3}$
}

\begin{abstract}
RESUMO: OBJETIVO: avaliar os danos relacionados ao trabalho entre técnicos administrativos de uma instituição pública de ensino superior na Paraíba. MÉTODO: A pesquisa teve um caráter descritivo com a participação de 91 servidores técnicosadministrativos efetivos, de ambos os sexos, com atividade laboral executada na Universidade Estadual da Paraíba. A amostra foi formada pelo maior número possível de participantes que foram incluídos conforme a acessibilidade e disponibilidade em colaborar com a pesquisa. Os instrumentos utilizados para realização da pesquisa foram a Ficha Sócioeconômica, Escala de Avaliação dos Danos Relacionados ao Trabalho (EADRT), uma das escalas do Inventário sobre Trabalho e Riscos de Adoecimento - ITRA, além do Questionário Nórdico de Sintomas Osteomusculares - QNSO. RESULTADOS: No trabalho dos técnicosadministrativos existem vivências de prazer, mas, também de sofrimento, onde, o prazer está relacionado as relações sócio profissionais e nos fatores sociais e psicológicos. Já o sofrimento surge principalmente nos danos físicos e distúrbios biológicos. CONCLUSÃO: De acordo com os resultados encontrados percebe-se a importância de um olhar voltado para a saúde do trabalhador, onde, os riscos de adoecimento são visíveis, o que gera uma responsabilidade para o melhor cuidado da saúde dos técnicos-administrativos da referida universidade.
\end{abstract}

Palavras chave: Categoria de Trabalhadores. Riscos Ocupacionais. Trabalho.

\footnotetext{
${ }^{1}$ Graduanda de Fisioterapia pela Universidade Estadual da Paraíba - UEPB, Campus I, Campina Grande, PB.

${ }^{2}$ Doutora, Docente da Universidade Estadual da Paraíba- UEPB, Campus I, Campina Grande, PB.

${ }^{3}$ Doutora, Docente da Universidade Estadual da Paraíba - UEPB, Campus I, Campina Grande, PB.
} 
ABSTRACT: OBJECTIVE: to evaluate the work-related damages among administrative technicians of a public institution of higher education in Paraiba. METHOD: The research had a descriptive character with the participation of 91 effective technical-administrative servers, of both sexes, with work activity performed at the State University of Paraiba. The sample was formed by the largest possible number of participants who were included according to accessibility and availability to collaborate with the research. The instruments used to carry out the research were the Socio-Economic Form, Work-Related Damage Assessment Scale (EADRT) one of the scales of the Inventory on Work and Risks of IIIness - ITRA, in addition to the Nordic Questionnaire of Musculoskeletal Symptoms - QNSO. RESULTS: In the work of the administrative technicians there are experiences of pleasure, but also of suffering, where pleasure is related to socio-professional relationships and social and psychological factors. Onthe other hand, suffering arises mainly in physical damage and biological disorders. CONCLUSION: According to the results found, it is perceived the importance of a look at the health of the worker, where the risks of illness are visible, which generates a responsibility for the better health care of the administrative technicians of the said university.

Keywords: Occupational Risks. Work. Category of Workers. 


\section{INTRODUÇÃO}

O trabalho se apresenta como fundamental para existência humana. Neste sentido, podendo influenciar no bem-estar gerando sintomas que afetam a saúde. Não há como pensar em um mundo no trabalho e outro fora dele, afinal, trabalhadores são influenciados por cada contexto onde se encontram inseridos, seja laboral, familiar ou social. A saúde do trabalhador constitui tema imprescindível para discussão e destaque (SILVA; LIMAS; PEREIRA, 2016).

Devido aos estudos desenvolvidos durante anos por historiadores, filósofos e profissionais da área da saúde acerca da relação entre trabalho e doença, tem-se observado como os vários tipos de ocupações, como por exemplo, hospitalares, administrativos, policiais e etc. estão afetando a saúde das pessoas e desenvolvimento científico. Neste sentido, permitindo estabelecer medidas para tratar ou prevenir doenças ocupacionais. Diante da evolução da indústria, o trabalho torna-se mais dependente da técnica e, pobre em qualidade de movimentos, aumentando o número de acidentes e doenças profissionais. Certas atividades exigem dos trabalhadores a ação dos mesmos grupos musculares por meses ou anos, podendo conduzir ao desenvolvimento de lesões (SICOLI, 2011).

Os danos relacionados ao trabalho representam manifestações de efeitos nocivos à saúde, em consequência das exigências e vivências presentes na atividade laboral. Danos físicos são expostos na forma de manifestações como dores no corpo e distúrbios biológicos. Os danos psicológicos caracterizam-se por sentimentos negativos em relação a si mesmo e à vida no geral, já os danos sociais compreendem dificuldades nas relações sociais e familiares (PRESTES, 2016).

De acordo com o Ministério da Saúde, o termo saúde do trabalhador compreende as relações entre o trabalho e o processo saúde/doença, entendendo que a forma como os trabalhadores são inseridos no seu local de trabalho e como isso afeta decisivamente nas formas especificas de adoecer e morrer (BRASIL, 2018). Como determinantes da saúde do trabalhador estão os condicionantes 
sociais, econômicos, tecnológicos e organizacionais responsáveis pelas condições de vida e fatores de risco ocupacionais - físicos, químicos, biológicos, mecânicos, e aqueles decorrentes da organização laboral - presentes nos processos de trabalho (BRASIL, 2001).

Estudo sobre a relação saúde e trabalho na área pública brasileira têm dado a conhecer as condições de vida e trabalho as quais estão expostos os servidores públicos. Fazem parte deste grupo os Técnicos-Administrativos em Educação TAEs e os Docentes que são as duas categorias que compõem os quadros de servidores das universidades públicas do Brasil. Infelizmente as autoridades governamentais ainda se encontram ausentes quando o assunto é Segurança do Trabalho, doenças ocupacionais e qualidade de vida dos servidores públicos. Porém, essa falha apenas se torna elucidada quando trabalhadores ficam expostos a riscos ambientais e sofrem as consequências. Por isso, visualiza-se de suma importância que haja valorização do ser humano como um todo, visando atingir todas as áreas as quais estão inseridos e realizar procedimentos para garantir seus direitos individuais e coletivos, tendo como principal investimento a preservação da saúde e qualidade de vida (BRASIL, 2018).

Para melhoria na saúde do trabalhador, tem-se como principal objetivo a promoção emsaúde. Sendo desenvolvida por intermédio de ações de vigilância dos riscos presentes no ambiente de trabalho e agravos à saúde do trabalhador, como também, por meio da organização e prestação de assistência aos trabalhadores, compreendendo procedimentos de diagnóstico, tratamento e reabilitação dos mesmos (BRASIL, 2001). Diante do exposto, demonstra-se a importância dos servidores públicos serem assistidos e protegidos de seus direitos em virtude da vida organizacional ser capaz de provocar insegurança ou oferecer riscos à saúde (ANJOS, 2014).

A exposição das áreas corporais atingidas durante a execução das atividades laborais revelará um novo olhar para formatação de políticas institucionais de saúde do trabalhador. Neste contexto, o objetivou-se avaliar os danos relacionados ao trabalho entre técnicos-administrativos de uma instituição pública de ensino superior na Paraíba. 


\section{METODOLOGIA}

A metodologia da presente pesquisa possui caráter descritivo, pois visa tornar o fenômeno mais explícito e analisar sua ocorrência, estabelecendo relações entre as principais variáveis do estudo sem manipulá-las. A amostra foi composta a partir de uma estratégia acidental, definida como não-probabilística, sendo formada pelo maior número possível de participantes que vão sendo incluídos conforme a acessibilidade e disponibilidade em colaborar com a pesquisa. Tal acessibilidade foi mediada pelos chefes de cada setor, sendo os instrumentos preenchidos por todos os técnicos administrativos que aceitaram voluntariamente participar do estudo. Assim, participaram da pesquisa 91 servidores técnicos-administrativos.

A pesquisa foi realizada no Campus I, da Universidade Estadual da Paraíba localizada na Rua Baraúnas, n. 351, Bairro Universitário, em Campina Grande, Paraíba, com servidores técnicos-administrativos no próprio local de trabalho. Como critério de inclusão foram considerados servidores técnicos-administrativos efetivos, de ambos os sexos, com atividade laboral executada na UEPB, campus I. Nos critérios de exclusão estão servidores técnicos nãoefetivos, servidores afastados de suas funções ou cedidos para outras instituições e trabalhadores que não desempenham sua função na instituição.

Para coleta de dados foi aplicado Questionário Socioeconômico visando coletar informações relativas ao perfil biográfico e sócio ocupacional (idade, sexo, estado civil, nível de instrução escolar, número de filhos tempo de serviço, renda e afastamento do trabalho por problemas de saúde relacionados ao trabalho) a fim de caracterização da amostra.

Um dos instrumentos utilizados foi a Escala de Avaliação dos Danos Relacionados ao Trabalho (EADRT), a qual foi retirada do Inventário sobre Trabalho e Riscos de Adoecimento - ITRA. Esse instrumento foi construído e validado por Ferreira e Mendes (2003) em pesquisa nacional com auditores fiscais da Previdência Social Brasileira, sendo posteriormente adaptado e validado com outras amostras ocupacionais (MENDES; FERREIRA, 2007). Os autores recomendam seu 
uso para fins de diagnóstico ocupacional e pesquisas acadêmicas voltadas a melhoria da saúde e qualidade de vida do trabalhador.

A Escala de Avaliação dos Danos Relacionados ao Trabalho (EADRT), contem 29 itens que variam de 0 a 6 pontos, distribuídos em três fatores: F1) Danos Físicos, que avalia se os respondentes sentem dores corporais e distúrbios biológicos provocados pelo trabalho; F2) Danos Psíquicos, que verifica sentimentos negativos em relação a si mesmo e a vida em geral e; F3) Danos Sociais, que analisa sentimentos de isolamento e dificuldades nas relações familiares e sociais.

Outra ferramenta utilizada foi o Questionário Nórdico de Sintomas Osteomusculares - QNSO, instrumento validado e adaptado para língua portuguesa por Barros e Alexandre (2003). É formado por uma figura humana dividida em nove regiões anatômicas que compreende questões quanto à presença de dores musculoesqueléticas semanal e anual, incapacidade funcional e afastamento nos últimos 12 meses de atividades como trabalho ou lazer (CARVALHO ; ALEXANDRE, 2006; SILVA; SANTOS, 2010).

A coleta de dados teve início após a aprovação do Comitê de Ética da Universidade Estadual da Paraíba (UEPB) com parecer de aprovação: $n^{\circ} 3.124 .214$ como rege a resolução 466/2012 do Conselho Nacional de Saúde/MS. Assim, participaram da pesquisa os técnicos de ambos os sexos que estavam em pleno exercício da função e que aceitaram assinar o Termo de Consentimento Livre e Esclarecido (TCLE) em duas vias, ficando uma em posse do participante, e a outra, do (a) pesquisador (a).

Foi realizada a aplicação do questionário contendo o perfil socioeconômico, a Escala de Avaliação dos Danos Relacionados ao Trabalho (EADRT) e o Questionário Nórdico de Sintomas Osteomusculares (QNSO), no tempo conveniente ao técnico administrativo. Cada participante respondeu aos questionários no próprio local de trabalho. Antes de receber os questionários, todos foram informados sobre os objetivos e os aspectos éticos da pesquisa. Feito isto, era realizado o preenchimento do questionário e o pesquisador permanecia no local para esclarecer eventuais dúvidas. O tempo gasto para responder todas as questões era de aproximadamente 15 minutos. 
As respostas contidas nos questionários foram digitadas na forma de banco de dados no Excel e, em seguida, examinado a consistência interna dos fatores, calculada por meio do Alfa de Cronbach, onde o resultado da análise da EADRT foi de 0,94 sendo classificada como muito boa, atendendo aos critérios dos estudos, sendo possível utilizar análises estatísticas.

Para Mendes e Ferreira (2007) considera-se como resultado para a Escala de Avaliação dos Danos Relacionados ao Trabalho (EADRT) os seguintes valores: Acima de 3,7 = avaliação mais negativa, grave; Entre 2,3 e 3,69 = avaliação mais moderada, crítico; Abaixo de 2,29 = avaliação mais positiva, satisfatório. Também foram efetuadas as análises descritivas (média, desvio-padrão e porcentagem) para delinear o perfil socioeconômico da amostra e observar os indicadores descritivos da distribuição dos escores individuais obtidos em cada fator.

\section{RESULTADOS E DISCUSSÃO}

\section{Questionário socioeconômico}

Dos 91 técnicos-administrativos que responderam ao questionário foi observado oseguinte perfil socioeconômico: 
Tabela 1 - Perfil Socioeconômico dos técnicos-administrativos.

\begin{tabular}{|c|c|c|}
\hline VARIÁVEIS & $\mathbf{N}$ & $\%$ \\
\hline \multicolumn{3}{|l|}{ Sexo } \\
\hline Feminino & 42 & $46 \%$ \\
\hline Masculino & 49 & $54 \%$ \\
\hline \multicolumn{3}{|l|}{ Faixa Etária } \\
\hline 21 a 30 anos & 38 & $42 \%$ \\
\hline 31 a 40 anos & 45 & $49,4 \%$ \\
\hline 41 a 50 anos & 4 & $4,3 \%$ \\
\hline 51 a 60 anos & 3 & $3,3 \%$ \\
\hline 61 a 70 anos & 1 & $1 \%$ \\
\hline \multicolumn{3}{|l|}{ Formação Acadêmica } \\
\hline Ensino Superior incompleto & 14 & $15 \%$ \\
\hline Ensino Superior Completo & 30 & $33 \%$ \\
\hline Pós - Graduação & 47 & $52 \%$ \\
\hline \multicolumn{3}{|l|}{ Estado Civil } \\
\hline Casados & 33 & $36,3 \%$ \\
\hline Solteiros & 56 & $61,53 \%$ \\
\hline Divorciados & 2 & $2,2 \%$ \\
\hline \multicolumn{3}{|l|}{ Número de Filhos } \\
\hline Zero & 61 & $67 \%$ \\
\hline Um & 17 & $18,7 \%$ \\
\hline Dois & 9 & $10 \%$ \\
\hline Três & 3 & $3,3 \%$ \\
\hline Quatro & 1 & $1 \%$ \\
\hline \multicolumn{3}{|l|}{ Renda Mensal } \\
\hline Não declararam & 16 & $17,6 \%$ \\
\hline 1 - 2 salários mínimos & 6 & $7 \%$ \\
\hline 3 - 4 salários mínimos & 45 & $49,4 \%$ \\
\hline 5 - 6 salários mínimos & 23 & $25,3 \%$ \\
\hline Acima de 6 salários mínimos & 1 & $1 \%$ \\
\hline \multicolumn{3}{|c|}{ Tempo de Serviço na Instituição } \\
\hline $1-11$ meses & 33 & $36,3 \%$ \\
\hline $1-5$ anos & 18 & $19,8 \%$ \\
\hline $6-10$ anos & 27 & $29,6 \%$ \\
\hline $11-15$ anos & 9 & $10 \%$ \\
\hline $16-20$ anos & 0 & $0 \%$ \\
\hline $21-35$ anos & 3 & $3,3 \%$ \\
\hline & & \\
\hline \multicolumn{3}{|c|}{ Afastamento do trabalho por problemas de saúde relacionados ao trabalhe } \\
\hline Nunca & 83 & $91,2 \%$ \\
\hline $1-3$ vezes & 8 & $8,8 \%$ \\
\hline Mais de 3 vezes & 0 & $0 \%$ \\
\hline
\end{tabular}

Fonte: O autor (2019). 
A partir da análise dos dados, observou-se em linhas gerais existir população em estudo no fator sexo, pois, apesar da porcentagem maior ser a masculina, a população feminina não está tão a baixo, mostrando que a mulher vem tomando cada vezmais espaço no mercado de trabalho.

Quanto a idade dos servidores, tempo de serviço, número de filhos e estado civil foi observada uma grande quantidade de jovens adultos e adultos a baixo de 41 anos totalizando $91,4 \%$ dos pesquisados, como também $36,3 \%$ da população são solteiros, $67 \%$ não tem filhos e $36,3 \%$ estão a menos de um ano trabalhando na instituição, o que evidencia que as pessoas têm buscado cada vez mais cedo estabilidade financeira por meio de concurso público corroborando com trabalho publicado por Albrecht e Krawulski (2011) os quais afirmam que a maioria dos pesquisados almejantes ao ingresso no serviço público são jovens adultos no início de carreira profissional e que a busca pelo o setor público se deve a perspectivas salariais, segurança e estabilidade, e ainda as boas condições de trabalho, que são fatores de grande atrativo para os trabalhadores. Nesta pesquisa $75,7 \%$ dos pesquisados têm renda acimade três salários mínimos.

Em relação a formação acadêmica dos técnicos-administrativos, corroborando com estudo de Silva (2016), os dados mostram formação acadêmica superior ao segundo grau completo, requisito necessário para ingresso no cargo público, isso ocorre pois melhorias salariais se associam a capacitação profissional, o que é proposto pelo Ministério da Educação (MEC) definidas na Lei $n^{\circ} 11.091$, de 12 de janeiro de 2005 proposta nas Diretrizes para desenvolvimento do Servidor Público, como também no Plano de Carreira dos Cargos Técnico-Administrativos.

Quanto ao afastamento do trabalho por problemas de saúde relacionados ao trabalho, grande maioria afirmou nunca ter realizado afastamento por essa causa, porém, isso se deve a um número elevado de pessoas com tempo de serviço abaixo de um ano, corroborando com Freitas (2015) onde, assim como a presente pesquisa, a maioria dos entrevistados tem até 5 anos de tempo de serviço e afirmam que não obtiveram nenhum afastamento por problemas de saúde relacionado ao trabalho assim, os números de afastamentos por motivos de saúde não se enquadram em situação alarmante e é uma condição satisfatória quanto à saúde dos servidores. 


\section{Resultados da Escala de avaliação dos Danos Relacionados ao Trabalho (EADRT)}

A Tabela 2 apresenta as médias do primeiro fator da Escala de Avaliação dos Danos Relacionados ao Trabalho (EADRT) que avalia as disfunções físicas, psicológicas e sociais decorrentes do contexto de trabalho. Nessa escala, os itens estarão relacionados a situações muito graves relacionadas a saúde. Neste sentido, aparecendo itens em nível moderado já indica adoecimento (FERREIRA; MENDES, 2007).

Tabela 2 - Resultados das respostas dos técnicos-administrativos em relação a dorescorporais e distúrbios biológicos provocados pelo trabalho.

\begin{tabular}{|c|c|}
\hline ITENS & MÉDIAS \\
\hline Dores no corpo & 2,4 \\
\hline Dores nos braços & 1,9 \\
\hline Dor de cabeça & 3,1 \\
\hline Distúrbios respiratórios & 0,8 \\
\hline Distúrbios digestivos & 1,1 \\
\hline Dores nas costas & 1,0 \\
\hline Distúrbios auditivos & 0,7 \\
\hline Alterações do apetite & 1,4 \\
\hline Distúrbios na visão & 1,6 \\
\hline Alterações do sono & 2,4 \\
\hline Dores nas pernas & 1,8 \\
\hline Distúrbios circulatórios & 0,8 \\
\hline
\end{tabular}

Acima de 3,7 = avaliação mais negativa, grave; Entre 2,3 e 3,69 = avaliação mais moderada,crítico; Abaixo de 2,29 = avaliação mais positiva, satisfatório.

Fonte: O autor (2019).

Observado os dados da Tabela 2, nenhum item desse fator apresentou gravidade, porém, três itens foram considerados como críticos: "Dores no corpo" $(2,4)$, "Dor de cabeça" $(3,1)$ e "Alterações no sono" $(2,4)$. Os fatores que estão relacionados ao fato de passar muito tempo na mesma posição, sem realização de 
uma pausa ativa e relaxamento, aumentam a probabilidade de dor e surgimento de doenças ocupacionais. Como também níveis de estresse elevados a ponto de alterar o sono. Assim, de acordo com a classificação, já se caracteriza como quadro de adoecimento. Este resultado corrobora com Costa (2017) que avaliou a Qualidade de Vida no Trabalho, incluindo o fator saúde nas condições de trabalho, sendo avaliado na pesquisa como neutro/moderadamente, apresentando assim queixa em relação a saúde no ambiente de trabalho como a presente pesquisa.

O segundo fator da Escala de Avaliação dos Danos Relacionados ao Trabalho (EADRT) está representado na Tabela 3, versando acerca do comportamento do próprio trabalhador, podendo apresentar alguma alteração no humor devido as ocorrências nocontexto de trabalho.

Tabela 3 - Resultados das respostas dos técnicos-administrativos em relação aos danospsíquicos.

\section{ITENS}

Insensibilidade em relação aos colegas

Dificuldades nas relações fora do trabalho

Vontade de ficar sozinho

Conflitos nas relações familiares

Agressividade com os outros

Dificuldade com os amigos Impaciência com as pessoas em geral

\section{MÉDIAS}

1,0

1,1

1,9

0,8

0,8

0,8

1,8

Média Total

1,1

Acima de 3,7 = avaliação mais negativa, grave; Entre 2,3 e 3,69 = avaliação mais moderada,crítico; Abaixo de 2,29 = avaliação mais positiva, satisfatório.

Fonte: O autor (2019).

Em relação aos danos sobre o comportamento dos técnicos-administrativos apresentados na Tabela 3 todos foram satisfatórios. As maiores médias sendo: "conflitos nas relações familiares", "Agressividade com os outros" e Dificuldade com os amigos" obtiveramos mesmos resultados $(0,8)$, demonstrando que esses fatores não influenciaram no seu comportamento perante a família e sociedade. Corroborando com Costa (2017) que avaliou a Qualidade de Vida no Trabalho, e um dos itens trata acerca da integração social na organização, sendo classificado na 
pesquisa como bom, apresentando semelhança com a presente pesquisa onde o resultado do seguinte item foi satisfatório.

A Tabela 4 apresenta os resultados do último fator da escala em relação aos danos Psicológicos sofridos pelos técnicos-administrativos.

Tabela 4 - Resultados das respostas dos técnicos-administrativos em relação aos danosociais.

\begin{tabular}{lc}
\hline ITENS & MÉDIAS \\
\hline Amargura & 0,8 \\
Sensação de vazio & 0,8 \\
Sentimento de desamparo & 0,9 \\
Mau-humor & 2,1 \\
Vontade de desistir de tudo & 1,0 \\
Tristeza & 1,4 \\
Irritação com tudo & 1,4 \\
Sensação de abandono & 1,0 \\
Dúvida sobre a capacidade de fazer as tarefas & 1,2 \\
Solidão & 1,1 \\
\hline Média Total & $\mathbf{1 , 1}$ \\
\hline
\end{tabular}

Acima de 3,7 = avaliação mais negativa, grave; Entre 2,3 e 3,69 = avaliação mais moderada,crítico; Abaixo de 2,29 = avaliação mais positiva, satisfatório.

Fonte: O autor (2019).

No último fator apresentado na Tabela 4, todos os itens foram satisfatórios, porém o item "Mau-humor" apresentou a maior média $(2,1)$, ficando próximo de ser considerada como crítico. Os dois fatores com médias mais baixas foram: "Amargura" e "Sensação de vazio" $(0,8)$.

\section{Resultados do Questionário Nórdico de Sintomas Osteomusculares (QNSO)}

Quanto a prevalência de dor e/ou desconforto nos últimos 12 meses, todos responderam as nove questões relacionadas a queixa ou não de sintomas 
musculoesqueléticos em diferentes partes do corpo humano, totalizando 819 respostas, dessas 302 foram positivas (37\%) e 517 negativas (63\%).

Como cada resposta positiva ou negativa corresponde a uma determinada parte do corpo humano, todas as áreas tiveram registros de queixa obedecendo a seguinte ordem: região com mais respostas positivas foi a parte inferior das costas com $n=55(18,2 \%)$, seguido da parte superior das costas com $n=50(16,5 \%)$, pescoço com $n=44(14,6 \%)$, ombros com $n=41$ (13,6\%), punhos/mãos com $n=38$ $(12,5 \%)$, joelhos com $n=27(9 \%)$, tornozelos/pés $n=25(8,3 \%)$, quadril/coxas $n=13$ $(4,3 \%)$ e cotovelos $n=9(3 \%)$.

Tais resultados são devido a má postura sentada como também muito tempo na mesma postura gerando quadros de dor em toda a coluna, ocorrendo principalmente na coluna lombar devido a posição sentada. $O$ ideal consiste no trabalhador alternar a postura sentada e em pé.

Quanto a prevalência de dor e/ou desconforto nos últimos sete dias. Das 819 respostas, 118 foram positivas totalizando $14,4 \%$ da população, seguindo a ordem de queixas: parte inferior das costas $n=24(20,3 \%)$, parte superior das costas $n=23$ $(19,5 \%)$, pescoço $n=19(16,1 \%)$, ombros $n=17 \quad(14,4 \%)$, joelhos $n=12(10,2 \%)$, punhos/mãos $n=10(8,5 \%)$, tornozelos/pés $n=7(6 \%)$, quadril/coxas $n=4(3,4 \%)$ e cotovelos $n=2(1,7 \%)$. Corroborando com o estudo de Silva (2016), referenciado nesta pesquisa, apontando exatamente a mesma ordem das regiões do corpo humano mais afetada pelo trabalho.

Quanto ao impedimento de realizar atividades normais devido a dor e/ou desconforto em alguma das nove regiões apontadas pelo QNSO nos últimos 12 meses, das 819 respostas, 78 foram positivas $(9,5 \%)$ e 741 foram negativas (90,5\%).

E em relação a consulta a algum profissional da área de saúde nos últimos 12 meses devido a condição de dor e/ou desconforto em alguma região, das 819 respostas, 70 foram positivas $(8,5 \%)$ e 749 foram negativas $(91,5 \%)$, apresentando que, apesar de muitas queixas em regiões do corpo como coluna lombar, cervical e membros superiores, a maioria não busca tratamento em nenhuma das áreas de saúde, não ocorrendo prevenção, e, infelizmente, procurando o serviço de saúde quando o quadro já se encontra em um alto nível de agravo. 
Gráfico 1 - Respostas dos Técnicos-administrativos em relação as partes do corpo maisacometidas com dor e/ou desconforto.

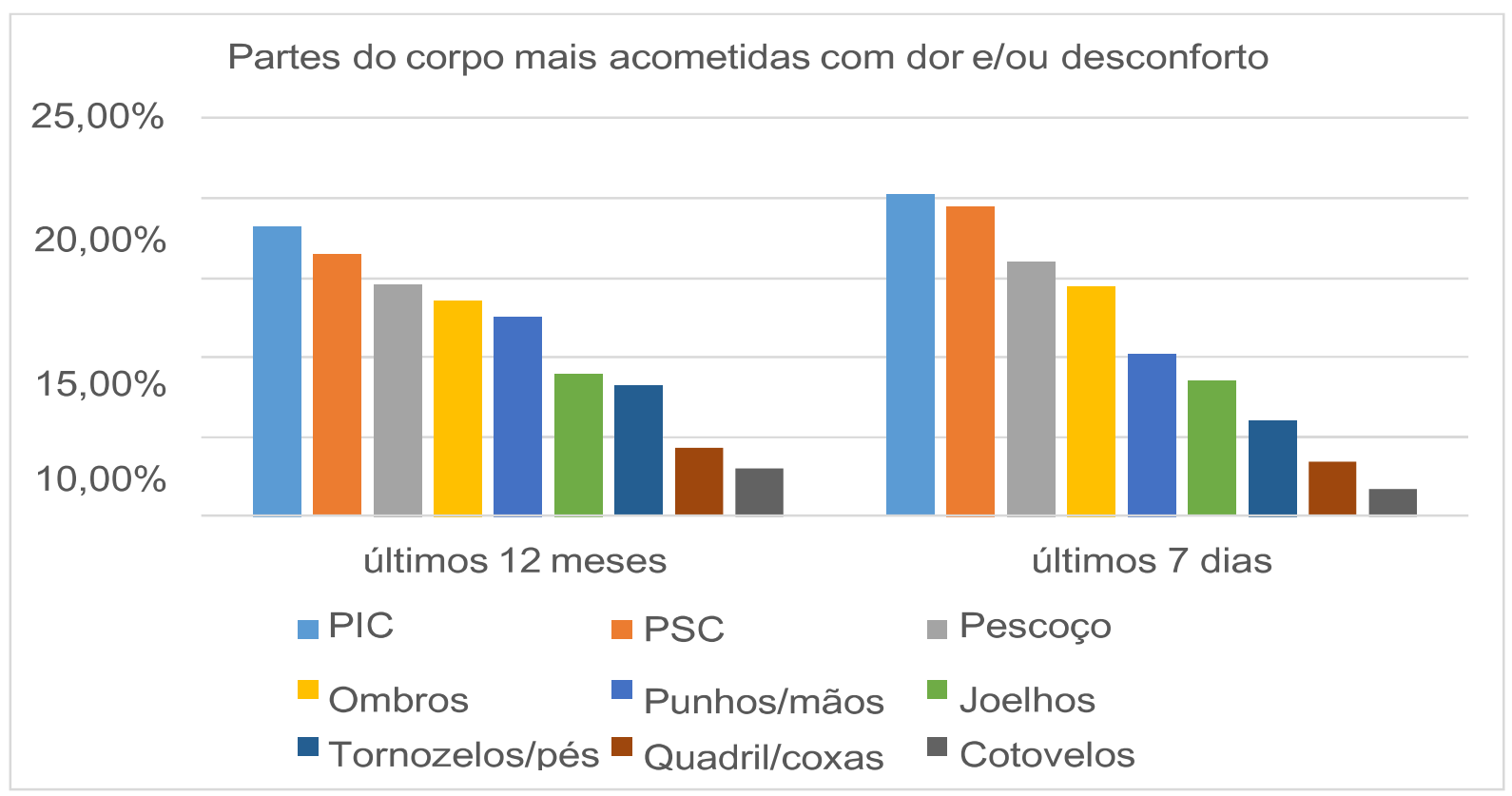

PIC: Parte Inferior das Costas; PSC: Parte Superior das Costas; Fonte: O autor (2019).

Assim, observado os resultados e visando melhoria na saúde desses trabalhadores, sugere-se investir em atenção e promoção da saúde, com palestras e informes acerca de mudanças para que os pontos negativos apresentados na pesquisa se tornem pontos de prazer. Faz-se necessário que o trabalhador entenda seus direitos, sendo necessário o conhecimento da Política Nacional de Saúde do Trabalhador e da Trabalhadora (PNSTT), a qual define os princípios, diretrizes e estratégias a serem observados pelas três esferas de gestão do Sistema Único de Saúde (SUS), no desenvolvimento das ações de promoção, vigilância, diagnóstico, tratamento, recuperação e reabilitação da saúde do trabalhador. Também se torna necessário a realização de Análise Ergonômica do Trabalho proposta pela Norma Regulamentadora 17 a qual irá avaliar a adaptação das condições de trabalho às características psicofisiológicas dos trabalhadores, alterando assim todo o mobiliário que estiver fora dos padrões descritos na norma. 


\section{CONCLUSÃO}

O perfil sócio demográfico ficou constituído, principalmente, de trabalhadores de ambos os sexos, entre 21 e 40 anos, pós-graduados, solteiros, sem filhos, que recebem de 3 a 4 salários mínimos, com tempo de serviço entre 1 mês e 5 anos relatando nunca ter ocorrido afastamento do trabalho decorrente de causa relacionada ao trabalho.

Quanto a Escala de avaliação dos Danos Relacionados ao Trabalho (EADRT) conclui-se que no trabalho dos técnicos-administrativos da Universidade Estadual da Paraíba (UEPB) existem vivências de prazer, mas, também de sofrimento, onde, o prazer está relacionado às relações sócio profissionais, fatores sociais e psicológicos. Já o sofrimento surge principalmente nos danos físicos e distúrbios biológicos como apresentado no QNSO, os quais se devem à falta de pausas, muito tempo sem alternar a postura e dor em toda a coluna emembros superiores.

Apesar dos resultados em relação aos danos físicos, no Questionário Nórdico de Sintomas Osteomusculares observa-se uma grande maioria dos técnicosadministrativos relatando não ter dor e/ou desconforto, isso é devido ao trabalho dos projetos de extensão do Núcleo de Educação e Atenção em Saúde que trabalham diretamente com técnicos- administrativos promovendo pausas ativas, massagem e momentos de relaxamento, proporcionando um ambiente saudável para os trabalhadores da referida universidade.

Assim, a partir dessa pesquisa percebe-se a importância de um olhar voltado para a saúde do trabalhador, onde, os riscos de adoecimento são visíveis, gerando responsabilidade para melhoria no cuidado da saúde dos técnicos-administrativos. Portanto, a partir dessa pesquisa percebe-se a importância de explanar acerca do tipo de trabalho, pois, os riscos de adoecimento entre técnicos-administrativos são visíveis e danosos. Outras pesquisas na área podem dar continuidade ao cuidado com a saúde do trabalhador, como também estudos qualitativos que trarão avaliações mais minuciosas. 


\section{REFERÊNCIAS BIBLIOGRÁFICAS}

ALBRECHT, Pricila Anny Tomachski; KRAWULSKI, Edite. Concurseiros e a busca por um emprego estável: reflexões sobre os motivos de ingresso no serviço público. Cadernos de Psicologia Social do Trabalho, [s.l.], v. 14, n. 2, p.211-226, 1 dez. 2011. Universidade de Sao Paulo Sistema Integrado de Bibliotecas - SIBiUSP.

ANJOS, D.L. Um olhar sobre a qualidade de vida no trabalho na área de qualidade de produto da empresa Porto Bello S/A: a luz da escala de Walton (1973). 2014. $134 \mathrm{f}$. Monografia (Bacharelado em Administração) - Universidade do Vale do Itajaí, Tijucas, 2014.

BARROS, E. N. C.; ALEXANDRE, N. M. C. Cross-cultural adaptation of the nordic musculoskeletal questionnaire. International Nursing Review, London, v. 50, n. 2, p. 101108, jun. 2003.

BRASIL. Ministério da Saúde. Saúde do Trabalhador. Caderno de atenção básica $n^{\circ} 5$. Brasília, 2018.

BRASIL. Ministério da Saúde. Doenças relacionadas ao trabalho: manual de procedimentos para os serviços de saúde. Brasília, 2001.

BRASIL. Ministério do Trabalho e Emprego. Nota Técnica n 060/2001. Ergonomia - indicação de postura a ser adotada na concepção de postos de trabalho. 2001.

CARVALHO A. J. F. P; ALEXANDRE N. M. C; Sintomas osteomusculares em professores do ensino fundamental. Revista Brasileira de fisioterapia, v 10, n.1,p.35-41, 2006.

FERREIRA, M. C.; MENDES, A. M. Inventário sobre trabalho e riscos de adoecimento ITRA: instrumento auxiliar de diagnóstico de indicadores críticos no trabalho. In: MENDES, A. M. (Org.). Psicodinâmica do trabalho: Teoria, método e pesquisas. São Paulo: Casa do Psicólogo, 2007.

FREITAS, K. S.; prazer e sofrimento no trabalho: um estudo com os servidores técnicos administrativos do ifto -campus palmas. 2015. 94.f. Dissertação (Mestrado em Gestão de Políticas Públicas) - Universidade Federal do Tocantins - UFTO, 2015.

ORGANIZAÇÃO MUNDIAL DE SAÚDE. Ambientes de trabalho saudáveis - um modelo para ação: para empregadores, trabalhadores, formuladores de política e profissionais. Brasília: Organização Mundial de Saúde, 2010. 26 p.

PRESTES, Francine Cassol. Danos à saúde dos trabalhadores de enfermagem em um serviço de hemodiálise. Revista Gaúcha de Enfermagem, [s.I.], v. 37, n. 1, p.1-7, 2016. FapUNIFESP (SciELO).

SILVA, P. da.; SANTOS, A. P. dos. Sintomas osteomusculares em docentes do ensino superior da faculdade de fisioterapia [Monografia]. Santos: Faculdade de Fisioterapia da Universidade Santa Cecília; 2010.

SILVA, Rosana Marques da; LIMA, Bruna Thays de; PEREIRA, Leonardo Santos. Contexto de trabalho e custo humano no trabalho: um estudo com trabalhadores portuários de transporte. Barbarói, [s.I.], n. 46, p.98-118, 9 mar. 2016. APESC - Associação Pro-Ensino em Santa Cruz do Sul.

SILVA, Ana Cláudia Colaço Lira. Fatores de Risco e Prevalência de Queixas 
Musculoesqueléticas entre Técnicos-administrativos em Educação: estudo realizado na Universidade Federal de Pernambuco. 2016. 169 f. Dissertação (Mestrado em Ergonomia) Universidade Federal de Pernambuco, 2016.

Sicoli, Elyseu. Uma abordagem sobre as Doenças Ocupacionais. 2011. 120 f. Tese (Doutorado em Ciências da Saúde, Eixo Temático: Medicina e Ciências Correlatas) - Faculdade de Medicina de São José do Rio Preto, 2011. 